\section{1-\$10.04 LIFESTYLE AS MARKER OF HEPATITIS C INFECTION IN HIV INFECTED MSM IN AMSTERDAM, THE NETHERLANDS}

doi:10.1136/sextrans-2011-050109.58

${ }^{1} \mathrm{~A}$ Matser, ${ }^{1} \mathrm{R}$ Geskus, ${ }^{1} \mathrm{~T}$ Heijman, ${ }^{1} \mathrm{~A}$ Urbanus, ${ }^{2} \mathrm{~J}$ Prins, ${ }^{1} \mathrm{H}$ de Vries, ${ }^{1} \mathrm{M}$ Prins, ${ }^{1} \mathrm{M} \mathrm{S}$ van der Loeff. 'Public Health Service, Amsterdam, Netherlands; ${ }^{2}$ Academic Medical Centre AMC, Amsterdam, Netherlands

Background Since 2000, hepatitis C virus (HCV) infection, associated with high risk sexual behaviour, has emerged as an STI among HIV infected MSM. We aimed to identify individual and networkrelated factors associated with HCV infection among HIV infected MSM.

Methods The study population was recruited at the STI outpatient clinic of the public health service and the HIV Treatment Centre of the Academic Medical Centre, Amsterdam, the Netherlands. Inclusion criteria were male gender, having had sexual contact with men in the previous 6 months, age $\geq 18$ years, and understanding of written Dutch or English. Recruitment occurred from July 2008 to August 2009. Participants were screened for chlamydia, gonorrhoea, syphilis, and hepatitis B. HIV testing was done using an opting-out strategy and HIV infected MSM were tested for the presence of HCV antibodies. Participants completed a questionnaire including demographics and detailed questions about sexual behaviour in selfdefined relationships with a steady partner and the most recent three other partners within the previous 6 months. Logistic regression analysis was used to identify factors associated with HCV infection

Results 850 HIV infected MSM reporting 2290 relationships were included in the study, of whom 108 were HCV antibody positive (overall prevalence $12.7 \%$ ). MSM who reported a history of injecting drug use $(n=3)$ were excluded. In multivariate analysis, independent risk factors for $\mathrm{HCV}$ infection were unprotected receptive anal intercourse (OR $1.6095 \%$ CI $1.00 \%$ to $2.57 \%$ ) and reporting group sex (OR 1.85 95\% CI 1.09\% to 3.15\%). Drug use (ie, XTC, GHB, or ketamine) was also associated with HCV (OR $2.2895 \%$ CI $1.33 \%$ to $3.92 \%$ ). There was significant interaction between fisting and self-described lifestyle type of MSM: the OR of non-fisting, leather/rubber/lycra type MSM was 4.22 (95\% CI $2.22 \%$ to $8.00 \%$ ); the OR of fisting non-leather/rubber/lycra type was 2.01 (95\% CI $1.00 \%$ to $4.04 \%$ ); and the OR of fisting leather/ rubber/lycra type was 2.71 (95\% CI $1.37 \%$ to $5.37 \%$ ), all compared to the reference group of non-fisting, non-leather/ rubber/lycra see Abstract O1-S10.04 table 1.

Conclusions Among HIV infected MSM in Amsterdam, HCV infection is associated with high risk sexual behaviour. HCV was common in identifiable risk groups of self-described 'leather' and 'rubber/lycra' type MSM. Ways should be sought to focus increased prevention efforts on these high risk groups.

\section{1-S10.05 COHORT STUDY TO DETERMINE SEXUALLY TRANSMITTED RECTAL INFECTIONS AMONG HIGH-RISK MEN WHO HAVE SEX WITH MEN IN INDIA}

doi:10.1136/sextrans-2011-050109.59

${ }^{1} \mathrm{M}$ Ranjan, ${ }^{1} \mathrm{~A}$ Das, ${ }^{1} \mathrm{P}$ Narayanan, ${ }^{1} \mathrm{~A}$ Gurung, ${ }^{2} \mathrm{~A}$ Risbud, ${ }^{2} \mathrm{R}$ Gangakhedkar. ${ }^{1} \mathrm{Family}$ Health International, New Delhi, India; ${ }^{2}$ National AIDS Research Institute, Pune, India

Background There are few studies on sexually transmitted infections (STIs) among men who have sex with men (MSM) in India to inform the development of appropriate strategies for STI control. This cohort study aimed to determine the prevalence and incidence of rectal gonorrhoea and chlamydia among high-risk MSM in response to standardised interventions in Avahan, the India AIDS Initiative of the Bill \& Melinda Gates Foundation. High-risk MSM are self-identified MSM who have large numbers of sex partners and sell sex or practice receptive anal sex.

Methods A cohort of 512 high-risk MSM attending STI clinics in two high HIV prevalence cities of India was recruited in 2008 and followed for four visits over a year. During each visit, rectal swabs were collected during proctoscopic examination and tested for Neisseria gonorrhoeae (GC) and Chlamydia trachomatis (CT) by Roche Amplicor PCR. Participants were provided risk reduction counselling and those with ano-rectal discharge syndrome received treatment as per the national guidelines. In addition, all participants received presumptive treatment for gonorrhoea and chlamydia at the baseline visit.

Results At baseline, rectal GC and/or CT prevalence was 14\% and remained consistent over the visits. Of those with laboratory confirmed infections, only $8.2 \%$ were clinically diagnosed with anorectal discharge syndrome, while the majority (91.8\%) did not have relevant symptoms or signs. Participants were followed for a total of 139.3 person years (median- 0.25 years, max -1.07 years) during which 1562 visits were made. During the study period, 127 incident

\section{Abstract 01-S10.05 Table 1 Correlates of GC/CT incidence}

\begin{tabular}{llll}
\hline S. No. & Characteristic & HR & p Value \\
\hline 1 & Age (up to 25 years) & 0.9 & 0.76 \\
2 & $\begin{array}{l}\text { Self-reported sexual identity of Panthi (insertive in anal sex) } \\
\text { compared to Kothi (receptive in anal sex) }\end{array}$ & 1.6 & 0.18 \\
3 & $\begin{array}{l}\text { Self-reported sexual identity of double decker (both insertive and } \\
\text { receptive) compared to Kothi }\end{array}$ & 0.9 & 0.63 \\
4 & Self-reported sexual identity of Panthi compared to double decker & 1.6 & 0.14 \\
5 & Engaged in commercial sex & 0.9 & 0.70 \\
6 & Sex work as main source of income & 1.0 & 0.90 \\
7 & New to commercial sex (<1 year) & 0.7 & 0.21 \\
8 & Self-reported condom use with male in last encounter & 2.3 & 0.06 \\
9 & Do not have regular partner & 1.0 & 0.96 \\
\hline
\end{tabular}

Abstract 01-S10.04 Table 1 Independent risk factors for HCV infection among 847 HIV infected MSM in Amsterdam

\begin{tabular}{|c|c|c|c|c|c|}
\hline & HCV prevalence & OR (95\% CI) & $\mathbf{p}$ & Adjusted OR (95\% CI) & $\mathbf{p}$ \\
\hline Non-fisting, non-leather/rubber/lycra type & $40 / 566(7.1 \%)$ & Ref. & $<0.001$ & Ref. & $<0.001$ \\
\hline Non-fisting, leather/rubber/lycra type & $25 / 99(25.3 \%)$ & $4.44(2.55$ to 7.74$)$ & & $4.22(2.22$ to 8.00$)$ & \\
\hline Fisting, non- leather/rubber/lycra type & $18 / 95(19.0 \%)$ & 3.07 (1.68 to 5.63$)$ & & $2.01(1.00$ to 4.04$)$ & \\
\hline Fisting, leather/rubber/lycra type & $24 / 87(27.6 \%)$ & $5.01(2.83$ to 8.85$)$ & & $2.71(1.37$ to 5.37$)$ & \\
\hline Drug use (XTC, GHB, ketamine) & 73 / 333 (21.9\%) & $3.96(2.57$ to 6.12$)$ & $<0.001$ & $2.28(1.33$ to 3.92$)$ & 0.002 \\
\hline Receptive unprotected anal intercourse & 58 / $277(21.0 \%)$ & $2.47(1.62$ to 3.77$)$ & $<0.001$ & $1.60(1.00$ to 2.57$)$ & 0.048 \\
\hline Group sex & 70 / $339(20.7 \%)$ & 3.31 (2.16 to 5.07$)$ & $<0.001$ & $1.85(1.09$ to 3.15$)$ & 0.022 \\
\hline Syphilis coinfection & $7 / 28(25.0 \%)$ & $2.02(0.83$ to 4.88$)$ & 0.141 & $2.83(1.01$ to 7.91$)$ & 0.046 \\
\hline
\end{tabular}


infections of rectal GC and CT were found giving an incidence rate of 91.2 per 100 person years. The incidence rate was highest when consecutive visits were within 30 days $(241.8$ per 100 person years, HR 0.83, $\mathrm{p}=0.00$ ). There were no significant associations of incidence of rectal gonorrhoea and chlamydia with demographic and behavioural characteristics (Abstract O1-S10.05 table 1).

Conclusions A high prevalence of asymptomatic rectal gonorhoea and chlamydia was observed along with a high incidence rate of these infections among high-risk MSM in India. The persistence of high prevalence of asymptomatic infections remaining even after presumptive treatment at the first visit calls for a review of the periodicity of presumptive treatment in similar clusters of MSM. Consistent condom use and partner treatment need to be reemphasised.

\section{1-S10.06 HIV/STI PREVALENCE AMONG MEN WHO HAVE SEX WITH MEN IN 4 CITIES, CHINA AND ASSOCIATED RISK FACTORS FOR HIV INFECTION}

doi:10.1136/sextrans-2011-050109.60

X Chen, N Jiang, B Wang. National Center for STD Control, China CDC, Nanjing, China

Objectives The data on STIs control and HIV prevention is limited among MSM population. To examine STIs control strategies for HIV prevention in a community-based cohort of HIV-negative MSM, community intervention trail was conducted between 2009 and 2011. This report mainly discussed baseline survey results.

Methods This study was conducted in MSM community of four mid-sized cities from Jul. to Sep. 2009. All participants were recruited through venue-based recruitment, complemented by peer referral using snowball method, Questionnaire were completed in STD clinics or VCT centers. Blood samples were collected for HIV, syphilis and HSV-2 tests, and urine sample for CT/NG PCR tests. Results $35.3 \%$ participants self-identified as homosexual and $44.7 \%$ bisexual. The most popular way to seek male sexual partners was internet (38.4\%). $15.9 \%$ of participants had provided or acquired sex services with male, while $19.1 \%$ of respondents reported unprotected anal intercourse (UAI) in the last sex services. 3.8\% of participants had experienced sadism \& masochism(SM). In past 6 months, $80.8 \%$ of participants had anal sex with man and $29.0 \%$ reported UAI during the last intercourse. $38.5 \%$ of participants reported having had sex with woman and only $45.2 \%$ of those reported using condom during the last intercourse with woman. $18.0 \%$ of participants involved in commercial sex services had taken drugs such as methamphetamine, Ketamine and MDMA.10.6\% of participants was HIV infection. $34.4 \%$ of participants is TP-ELISA positive results indicated a history of syphilis infection, and $20.9 \%$ were both positive results of ELISA and TRUST indicated active syphilis. $3.0 \%$ of participants were tested as NG infection, $6.8 \%$ was CT infected, and $16.2 \%$ were HSV-2 infected. Significant factors associated with HIV infection were self-reported STD infection history $[\mathrm{AOR}=2.1,95 \% \mathrm{CI}$ : $1.29 \%$ to $4.26 \%]$, syphilis infection $[\mathrm{AOR}=2.70,95 \% \mathrm{CI}: 1.81 \%$ to $4.04 \%]$, and HSV-2 infection [AOR $=3.07,95 \% \mathrm{CI}: 2.09 \%$ to $4.50 \%$ ].

Conclusions MSM have been potential bridge-population for HIV/ STIs from most-at-risk population to general population. Intervention activities should target the internet, sexual social networks, and certain subpopulations such as those taking drugs in commercial sex services or infected with STIs. Friendly and high-quality STIs service should reach to MSM who do not attend STD clinics. Campaigns are urgent not only to boost individual condom use but also to create culture for condom use in MSM community.

\section{Epidemiology oral session 11: Various topics} of special interest

\section{1-S11.01 TIME EVOLUTION OF THE FRACTION OF NEW HIV INFECTIONS DUE TO PRIMARY INFECTION AMONG HIGH RISK GROUPS IN SOUTHERN INDIA}

doi:10.1136/sextrans-2011-050109.6

${ }^{1} \mathrm{M}$ Pickles, ${ }^{1} \mathrm{M} \mathrm{C}$ Boily, ${ }^{2} \mathrm{P}$ Vickerman, ${ }^{3} \mathrm{~B} \mathrm{M}$ Ramesh, ${ }^{4} \mathrm{R}$ Washington, ${ }^{5} \mathrm{~K}$ Deering, ${ }^{3} \mathrm{~S}$ Verma, ${ }^{6} \mathrm{~J}$ Blanchard, ${ }^{6} \mathrm{~S}$ Moses, ${ }^{7} \mathrm{M}$ Alary. ${ }^{1}$ Imperial College London, UK; ${ }^{2}$ LSHTM, UK; ${ }^{3}$ Karnataka Health Promotion Trust, Bangalore, India; ${ }^{4}$ St John's Research Institute, Bangalore India; ${ }^{5}$ University of British Columbia, Canada; ${ }^{6}$ University of Manitoba, Winnipeg, Canada; ${ }^{7}$ Centre hospitalier affilié universitaire de Québec, Quebec, Canada

Background HIV infectivity is considerably higher during primary infection (PI: first few months after infection). PI is believed to be an important biological driver of HIV transmission at the population level, which has implication for prevention. We aimed to estimate the contribution of PI to HIV spread among high-risk groups in southern India.

Methods We used incidence predictions from a bespoke dynamical model of HIV transmission among FSW/clients parameterised and fitted to district-specific behavioural data and HIV/STI prevalence estimates, within a Bayesian framework. Multiple rounds of crosssectional survey data from among FSW/clients of Mysore and Belgaum, carried out as part of the evaluation of Avahan, the India AIDS initiative, were used to inform plausible parameter ranges and estimate HIV prevalence. Assumed risk of HIV infection (RRHIV/ PI) during PI was elevated by 4.5- to 19-fold compared to asymptomatic infections (based on systematic review) and lasted for 3 to 6 months. The annual fraction of new HIV infections (AF) due to primary infection was estimated over time from the 196 and 796 posterior parameter sets fitting Mysore and Belgaum data, respectively.

Results The median AF (overall: clients+FSW) declined from $57.3 \%$ ( $\min =34, \max =82$ ) \% in 1987 (assumed start of epidemic) to $49 \%$ $(29,70) \%$ in $2000,43.7 \%(25,65) \%$ in $2004,23.8 \% / 24.3 \%(10 / 10,42 /$ $43) \%$ in 2010/2025 for Mysore (Abstract O1-S11.01 figure 1). Similar AF estimates were obtained in Belgaum and for FSWs and clients separately in both districts. The univariate association between AF and RRHIV/PI declined slightly over time (Abstract O1-S11.01 figure 1). In multivariate regression analysis, RRHIV/PI (slope $=0.027$ ), duration of the whole infectiousness period (slope $=-0.014$ ) and duration of primary infection (slope $=0.587$ ) were significantly associated with AF early in the epidemic (1988) $(\mathrm{R} 2=0.833$, $\mathrm{p}$ value $<0.001)$. However, the AF in 2025 was also associated with number of years selling sex by street-based FSW (slope $=-0.020, p$ value $=0.042$ ) and number of years buying sex by high-activity clients (slope $=-0.004, p$ value $=0.055)(R 2=0.684)$.

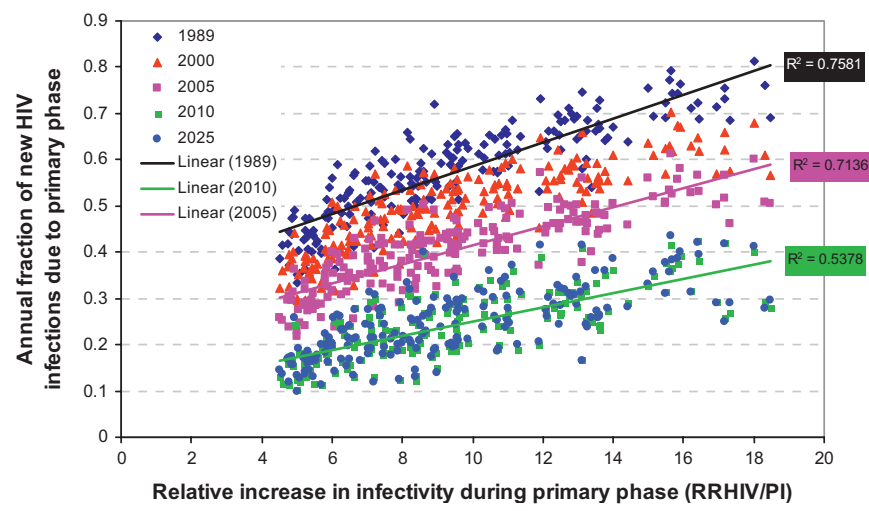

Abstract 01-S11.01 Figure 1 FSW and clients combined Mysore. 